\title{
Theory of anomalous dispersion in porous media
}

\author{
Stephen S. L. Peppin
}

\begin{abstract}
A theory of anomalous dispersion in porous media is developed by taking the high Peclet number limit of the equations describing cross-diffusion in concentrated suspensions. This leads to a modified advection-dispersion equation containing a reflection coefficient in the advection term. The reflection coefficient accounts for the effect of matrix heterogeneity, velocity fluctuations and pore-scale eddies on solute transport, and can be determined as a function of the relative tracer concentration from a single measured breakthrough curve. Once the reflection coefficient is known the model predicts anomalous breakthrough curves at various positions and flowrates within the porous medium. In addition the model uses the actual fluid velocity, in contrast to the classical advection-dispersion model in which the velocity is treated as an adjustable parameter.
\end{abstract}

\section{Introduction}

Tracer dispersion in porous media is often found to be anomalous, exhibiting experimental breakthrough curves with sharper fronts and longer tails than predicted by the classical advectiondispersion equation (ADE) [1-4]. Extensive experimentation and numerical modelling has shown that the origin of the anomalous dispersion in non-reactive systems is physical heterogeneity in the porous medium and local fluctuations in the fluid velocity [3,5-9]. Several approaches have been developed to simulate anomalous dispersion, including the continuous time random walk (CTRW) [3,10], fractional Brownian motion [11], percolation [12] and stochastic advection-dispersion models $[2,13,14]$. These methods are able to reproduce the anomalous shape of the measured breakthrough curves, and the CTRW method has been used extensively to model anomalous dispersion in a variety of systems $[1,15]$. However, the parameters involved do not always have a clear physical interpretation and the models are non-deterministic and non-local making them difficult to couple with classical Eulerian transport modelling codes [16]. In this work an alternative approach is taken in which dispersion within a porous medium is treated as a type of high Peclet number cross-diffusion within a particulate suspension near the close-packed/porous-medium limit. The approach leads to a coupling effect between the solute dispersion and the Darcy flow, in which the advection-dispersion equation contains a viscous reflection coefficient $\sigma$ in the advection term. Allowing the reflection coefficient to depend on the relative tracer concentration leads to very good agreement with experimental anomalous breakthrough curves. The approach maintains the classical deterministic structure of the ADE model, at the cost of introducing a nonlinearity in the advection term.

In Section 2 the ADE model is briefly reviewed and compared to an experimental anomalous breakthough curve on a homogeneous porous medium composed of sand particles. In Section 3 the cross-diffusion model is developed and the viscous reflection coefficient is determined from the measured breakthrough curve. In Section 4 the model is applied to several laboratory systems and to a river dispersion experiment, and Section 5 contains a discussion of the model and its relation to the stochastic approach to dispersion.

For God so loved the world, that he gave his only begotten Son, that whosoever believeth in him should not perish, but have everlasting life. For God sent not his Son into the world to condemn the world; but that the world through him might be saved. John 3:16,17 


\section{Advection-dispersion model}

In the ADE model the solute dispersion flux in a rigid stationary porous medium is given by Fick's law in the form

$$
\boldsymbol{J}=c \boldsymbol{q}-n D \boldsymbol{\nabla} c,
$$

where $\boldsymbol{J}=n c \boldsymbol{v}_{2}$ is the solute mass flux, $n$ is the porosity, $c$ is the solute concentration per unit volume of pore fluid, $\boldsymbol{v}_{2}$ is the average solute velocity, $\boldsymbol{q}=n \boldsymbol{v}$ is the Darcy flux of pore fluid moving at average velocity $\boldsymbol{v}$ and $D$ is the solute dispersion tensor $[17,18]$. At high flowrates the dispersion coefficient depends on the fluid velocity and is often written (for one dimensional systems) in the form

$$
D=D_{0}+\alpha_{d} v,
$$

where $D_{0}$ is the molecular diffusion coefficient and $\alpha_{d}$ is the dispersivity [17]. In heterogeneous porous media the dispersion coefficient may also depend on the lengthscale of the flow $[3,5,19]$. The fluid flux $\boldsymbol{q}$ is determined in the ADE model by Darcy's law

$$
\boldsymbol{q}=-\frac{k}{\eta} \nabla p
$$

where $p$ is the pore pressure, $k$ is the permeability and $\eta$ the fluid viscosity. Combining (1) with the solute mass conservation equation,

$$
\frac{\partial n c}{\partial t}+\nabla \cdot n c \boldsymbol{v}_{2}=0,
$$

leads to the classical advection-dispersion equation [17]

$$
\frac{\partial n c}{\partial t}+\nabla \cdot c \boldsymbol{q}=\boldsymbol{\nabla} \cdot n D \boldsymbol{\nabla} c,
$$

or, for an incompressible fluid $(\boldsymbol{\nabla} \cdot \boldsymbol{v}=0)$ in 1 dimension with constant $n$,

$$
\frac{\partial c}{\partial t}+v \frac{\partial c}{\partial x}=\frac{\partial}{\partial x} D \frac{\partial c}{\partial x} .
$$

For a solute flux $c_{0} v$ injected at $x=0$ into a fluid-saturated porous medium the boundary and initial conditions are of the form $[20,21]$

$$
c(0, t) v-D \frac{\partial c}{\partial x}=c_{0} v, \quad c(\infty, t)=0 \quad \text { and } \quad c(x, 0)=0 .
$$

For constant $D$ equations (6) and (7) have an analytical solution which at large Peclet numbers takes the form

$$
c(x, t)=c_{0} \frac{1}{2} \operatorname{erfc}\left(\frac{x-v t}{2 \sqrt{D t}}\right),
$$

where $\operatorname{erfc}(z)$ is the complementary error function [20,22]. Equation (8) assumes that the Peclet number $P e=v L / D \gg 1$, where $L$ is the position along the soil column at which the breakthrough curve is measured $[20,21] .^{1}$

\subsection{Breakdown of the ADE model}

Figure $1 a$ shows experimental data obtained by Cortis et al. [23] during injection of an $\mathrm{NaCl}$ solution into a porous medium (porosity $n=0.32$ ) composed of $0.23 \mathrm{~mm}$ diameter sand particles packed within a flowcell of length $L=0.20 \mathrm{~m}$ and cross-sectional area $A=5.0 \times 10^{-4} \mathrm{~m}^{2}$. In the experiment water was initially flowing through the porous medium with fluid velocity $v=Q / n A=6.0 \mathrm{~m} /$ day, where $Q=6.7 \times 10^{-7} \mathrm{~m}^{3} / \mathrm{s}$ was the measured fluid volume flux [23]. At $t=0$ the concentration

\footnotetext{
${ }^{1}$ In some experiments the concentrations measured are local volume-averaged [5] while in others flux-averaged concentrations are measured at the outlet reservoir [6]. As noted by van Genuchten and Parker [20] at low Peclet numbers the two experimental methods can give different results. In this work it is assumed that $P e \gg 1$ in which case it is not necessary to distinguish between flux-averaged and volume-averaged concentration measures [20,21].
} 

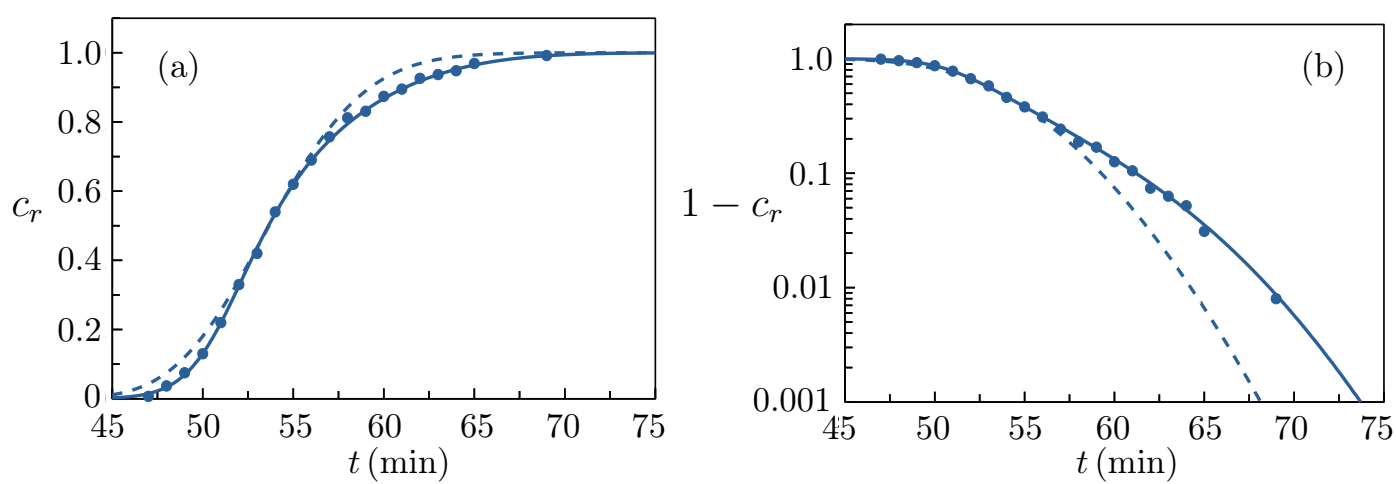

Figure 1: (a) Breakthrough curve of $c_{r}=c / c_{0}$ vs $t$ for $\mathrm{NaCl}$ dispersion within a column of sand $(L=0.20 \mathrm{~m})$. The data (circles) are from Cortis et al. [23]. The dashed curve is from the ADE model (8); the solid curve is from the generalized Burgers equation (16). (b) The same data plotted as $1-c_{r}$ versus $t$ on a log scale showing the long-time tail.

in the reservoir at $x=0$ was changed to $c_{0}=0.5 \mathrm{~g} / \mathrm{L}$. The outlet concentration at $x=L$ was measured as a function of time, with the data for the relative concentration $c_{r}=c / c_{0}$ shown in figure $1 a$. The dashed curve is the ADE solution (8) with best-fit parameters $v=5.3 \mathrm{~m} /$ day and $D=3.2 \times 10^{-3} \mathrm{~m}^{2} /$ day. Figure $1 b$ is a plot of $1-c / c_{0}$ vs $t$ on a $\log$ scale, showing the late-time tail. The ADE model is not able to reproduce the shape of the tail, nor the relatively sharp increase in concentration at early times. Furthermore, the best-fit fluid velocity is significantly smaller than the measured velocity. As has been demonstrated experimentally [6] and via numerical simulations $[3,7,8]$, the reason for the breakdown of the ADE model is local velocity fluctuations generated on the pore scale by the heterogeneous nature of the porous matrix. In order to account for such effects within a continuum mechanics framework, in the next section a model is developed that couples the diffusion flux $\boldsymbol{J}$ with the fluid flux $\boldsymbol{q}$.

\section{Coupled advection-dispersion model}

In this section a model of dispersion in porous media is developed by starting from the equations governing cross diffusion in concentrated suspensions. The approach is a continuation of previous work [24-28], but is modified here by taking the high-Peclet number limit. From nonequilibrium thermodynamics the flux equations of the solid matrix particles (component 1) and the solute particles (component 2) within a fluid can be written as

$$
\begin{aligned}
& \boldsymbol{J}_{1}=-L_{11} \boldsymbol{\nabla} \mu_{1}-L_{12} \boldsymbol{\nabla} \mu_{2}, \\
& \boldsymbol{J}_{2}=-L_{21} \boldsymbol{\nabla} \mu_{1}-L_{22} \boldsymbol{\nabla} \mu_{2},
\end{aligned}
$$

where $\boldsymbol{J}_{i}=c_{i}\left(\boldsymbol{v}_{i}-\boldsymbol{v}_{v}\right)$ is the flux of component $i$ at concentration $c_{i}$ (mass of particles per unit mixture volume) moving at speed $\boldsymbol{v}_{i}$ relative to the volume-average velocity $\boldsymbol{v}_{v}$ of the mixture; $\mu_{i}$ is the effective chemical potential per unit mass of $i$; and the $L_{i j}$ are phenomenological coefficients $[27,29,30]$. For inert particles within an incompressible fluid the volume-average velocity is $\boldsymbol{v}_{v}=n \boldsymbol{v}+(1-n) \boldsymbol{v}_{1}$, where $n$ is the porosity, $\boldsymbol{v}$ is the pore fluid velocity and $\boldsymbol{v}_{1}$ is the velocity of the particles making up the porous matrix.

By considering local equilibrium of a volume element within the system, the chemical potential gradients in (9) can be written in terms of the Darcy pore pressure $p$ and solute osmotic pressure $\pi \mathrm{as}^{2}$

$$
c_{1} \nabla \mu_{1}=-\nabla p+(1-n) \nabla \pi,
$$

\footnotetext{
${ }^{2}$ More generally, $c_{1} \nabla \mu_{1}=-\nabla p+(1-\alpha) \nabla \pi$ and $c_{2} \nabla \mu_{2}=\alpha \nabla \pi$, where $\alpha=c_{2} / c$ is the equilibrium partition coefficient of the solute between the pore space and the bulk fluid [27]. For molecular solutes in uncharged porous media, $\alpha$ is equal to the porosity $n$.
} 


$$
c_{2} \nabla \mu_{2}=n \boldsymbol{\nabla} \pi .
$$

At constant temperature the osmotic pressure gradient in an incompressible fluid can be written as

$$
\nabla \pi=\pi_{c} \nabla c
$$

where $\pi_{c}=(\partial \pi / \partial c)_{T, p}$ and $c=c_{2} / n$ is the tracer concentration per unit volume of pore fluid. Equations (9)-(11) assume that the length scale of any heterogeneity in the system is much smaller than the scale over which measurements are taken, so that the macroscopic system can be subdivided into relatively homogeneous volume elements similar to Bear's representative elementary volumes (REV) [17]. In very heterogeneous systems it may not be possible to define REVs in which case mesoscale models that explicitly treat each heterogeneity may be necessary $[31,32]$.

Combining (9), (10) and (11) leads after some rearrangement to the equations

$$
\begin{gathered}
\boldsymbol{q}=-\frac{k}{\eta}\left(\boldsymbol{\nabla} p-\sigma_{d} \boldsymbol{\nabla} \pi\right), \\
\boldsymbol{J}=c\left(1-\sigma_{v}\right) \boldsymbol{q}-n D \boldsymbol{\nabla} c,
\end{gathered}
$$

where $\boldsymbol{q}=\boldsymbol{v}_{v}-\boldsymbol{v}_{1}=n\left(\boldsymbol{v}-\boldsymbol{v}_{1}\right)=-\boldsymbol{J}_{1} / c_{1}$ is the Darcy flux of the pore fluid relative to the host matrix and $\boldsymbol{J}=n c\left(\boldsymbol{v}_{2}-\boldsymbol{v}_{1}\right)=\boldsymbol{J}_{2}-n c \boldsymbol{q}$ is the solute flux relative to $\boldsymbol{v}_{1}$. In equations (12) $k$ is the permeability of the host matrix, $\eta$ is the fluid viscosity, $\sigma_{d}$ is the osmotic reflection coefficient, $\sigma_{v}$ is the viscous reflection coefficient and $D$ is the tracer dispersion coefficient. In terms of the $L_{i j}$ these coefficients are defined as

$$
\frac{k}{\eta}=\frac{L_{11}}{c_{1}^{2}}, \quad \sigma_{d}=1-n+\frac{L_{12} c_{1}}{L_{11} c}, \quad \sigma_{v}=1-n+\frac{L_{21} c_{1}}{L_{11} c}
$$

and

$$
D=\frac{\pi_{c}}{n c}\left(L_{22}-\frac{L_{12} L_{21}}{L_{11}}\right) .
$$

Equations (12) are similar in form to the equations of membrane transport [33-36], but have here been derived in the context of cross-diffusion in concentrated suspensions and porous media.

\subsection{High Peclet number limit}

The reflection coefficients $\sigma_{d}$ and $\sigma_{v}$ in (12) are consequences of the coupling between the Darcy flow $\boldsymbol{q}$ and the tracer dispersion flux $\boldsymbol{J}$. When the reflection coefficients are zero equations (12a) and (12b) reduce to the traditional ADE model (1) and (3). Batchelor [29] has shown that for spherical particles in low Peclet number flows the Onsager reciprocal relation $L_{12}=L_{21}$ is valid, and $(13 a)$ then gives $\sigma_{d}=\sigma_{v}$. At high Peclet numbers, however, the Onsager relation no longer holds [29]. Here it is assumed that in high $P e$ flows the osmotic reflection coefficient is negligible $\left(\sigma_{d} \boldsymbol{\nabla} \pi \ll \nabla p\right)$ while the viscous reflection coefficient is relatively large $\left(\sigma_{v} \gg \sigma_{d}\right)$, so that equations (12) take the form

$$
\begin{gathered}
\boldsymbol{q}=-\frac{k}{\eta} \boldsymbol{\nabla} p, \\
\boldsymbol{J}=c(1-\sigma) \boldsymbol{q}-n D \boldsymbol{\nabla} c,
\end{gathered}
$$

where $\sigma=\sigma_{v}$.

For a rigid stationary porous medium $\boldsymbol{v}_{1}=0$, in which case $\boldsymbol{J}=n c \boldsymbol{v}_{2}$ and $\boldsymbol{q}=n \boldsymbol{v}$. Combining (14b) with (4) then gives a generalized advection-dispersion equation

$$
\frac{\partial n c}{\partial t}+\boldsymbol{\nabla} \cdot c(1-\sigma) \boldsymbol{q}=\boldsymbol{\nabla} \cdot n D \boldsymbol{\nabla} c .
$$

For an incompressible fluid $(\boldsymbol{\nabla} \cdot \boldsymbol{v}=0)$ in a 1D system with $n$ constant equation (15) becomes

$$
\frac{\partial c}{\partial t}+v \frac{\partial(1-\sigma) c}{\partial x}=\frac{\partial}{\partial x} D \frac{\partial c}{\partial x} .
$$


Similarly to the dispersion coefficient $D=D_{0}+\alpha_{d} v$, at high Peclet numbers the reflection coefficient $\sigma$ will depend on the average fluid velocity $v$ and the lengthscale over which the flow occurs. At larger lengthscales a solute plume experiences a wider range of velocity fluctuations, leading to entrainment effects and trapping by dead-zones and pore-scale eddies $[3,6]$. Here these effects are modelled by a constitutive equation of the form

$$
\sigma=\sigma_{0}+\alpha_{r} v,
$$

where $\sigma_{0}$ is a constant and $\alpha_{r}$ is the reflectivity of the porous matrix. The reflectivity is assumed to depend on the relative concentration of the solute plume $c_{r}=c / c_{0}$ via the polynomial

$$
\alpha_{r}=a\left(1-c_{r}\right)^{2}+b c_{r}^{2},
$$

where the constant $a$ governs the sharpness of the breakthrough curve near $c_{r}=0$, while $b$ accounts for the long-time tail in the region $c_{r} \rightarrow 1$. Other forms for $\alpha_{r}$ could also be employed, for example by including higher order terms as in Section 4.2, but (18) suffices to characterize the breakthrough curve for the relatively homogeneous porous medium in figure 1 . Because the viscous reflection coefficient $\sigma$ depends on $c$ via (17) and (18), equation (16) is in the form of a generalized Burgers equation [37-39].

\section{Results}

Equations (16)-(18) with boundary and initial conditions (7) were solved numerically using MatLab's pdepe function (see Supplementary Material for Matlab files). The solid curves in figure 1 show the solution using the measured fluid velocity $v=6.0 \mathrm{~m} /$ day and ADE dispersion coefficient $D=0.0032 \mathrm{~m}^{2} /$ day, along with the following parameters specifying the reflection coefficient, $\sigma_{0}=0.095, a=0.015$ days $/ \mathrm{m}$ and $b=0.0056$ days $/ \mathrm{m}$, yielding a good match to the data. In the nonlinear Burgers equation a diffuse front tends to sharpen with time because of the nonlinear advection term $[41,42]$. Here a similar effect occurs because of the dependence of the reflection coefficient $\sigma$ on the solute concentration, causing a sharpening of the profile as $c \rightarrow 0$ and broadening as $c \rightarrow c_{0}$. The dependence on $c$ represents an effective mapping of the dependence of $\sigma$ on position within the solute plume, accounting for the varying tendency of eddies and heterogeneity in the flow to 'reflect' the solute particles away from the average flow speed $v$ of the fluid. While the model introduces three new parameters, once the reflectivity $\alpha_{r}$ is known for a particular porous matrix, the model contains only 2 parameters $\left(\sigma_{0}\right.$ and $\left.D\right)$, the same number as for the ADE model $(v$ and $D)$. This is illustrated in the next section, where several breakthrough curves are simulated for a given porous medium at different positions and fluid velocities using a single unique reflectivity function.

\subsection{Dispersivity and reflectivity}

Figure 2 shows the effect of varying the fluid velocity $v$ on the breakthrough curves in a porous medium similar to that in figure 1. The data in figure 2 are from Levy and Berkowitz [6] on a porous medium of length $L=0.86 \mathrm{~m}$ and porosity $n=0.32$ composed of sand particles (diameter $0.53 \mathrm{~mm}$ ). Measured breakthrough curves were obtained using three different fluid velocities: $v=7.4 \mathrm{~m} /$ day (blue circles), $v=5.3 \mathrm{~m} /$ day (green triangles) and $v=3.6 \mathrm{~m} /$ day (red stars). The dashed curves in figure 2 are from the ADE model using a dispersivity of $\alpha_{d}=1.9 \mathrm{~mm}$ and best-fit fluid velocities $v=6.2 \mathrm{~m} /$ day (blue), $v=4.4 \mathrm{~m} /$ day (green) and $v=3.1 \mathrm{~m} /$ day (red). The solid curves are from equation (16) using the real fluid velocities, the ADE dispersivity and a single set of parameters to specify the reflectivity, $a=0.022$ days $/ \mathrm{m}$ and $b=0.0075$ days $/ \mathrm{m}$. The best-fit values of $\sigma_{0}$ were 0.12 (blue circles), 0.135 (green triangles) and 0.115 (red stars). As discussed further in Section $5, \sigma_{0}$ plays the role of an effective retardation factor that either slows $\left(\sigma_{0}>0\right)$ or enhances $\left(\sigma_{0}<0\right)$ the speed of the solute plume relative to the average fluid velocity. The positive values of $\sigma_{0}$ are consistent with the results of the ADE model in which the best-fit velocities are all slower than the measured fluid velocities.

The results in figure 2 suggest that once the reflectivity $\alpha_{r}$ is known for a given porous medium the breakthrough curves can be obtained at various flowrates $v$ and positions $L$ by fitting for only 

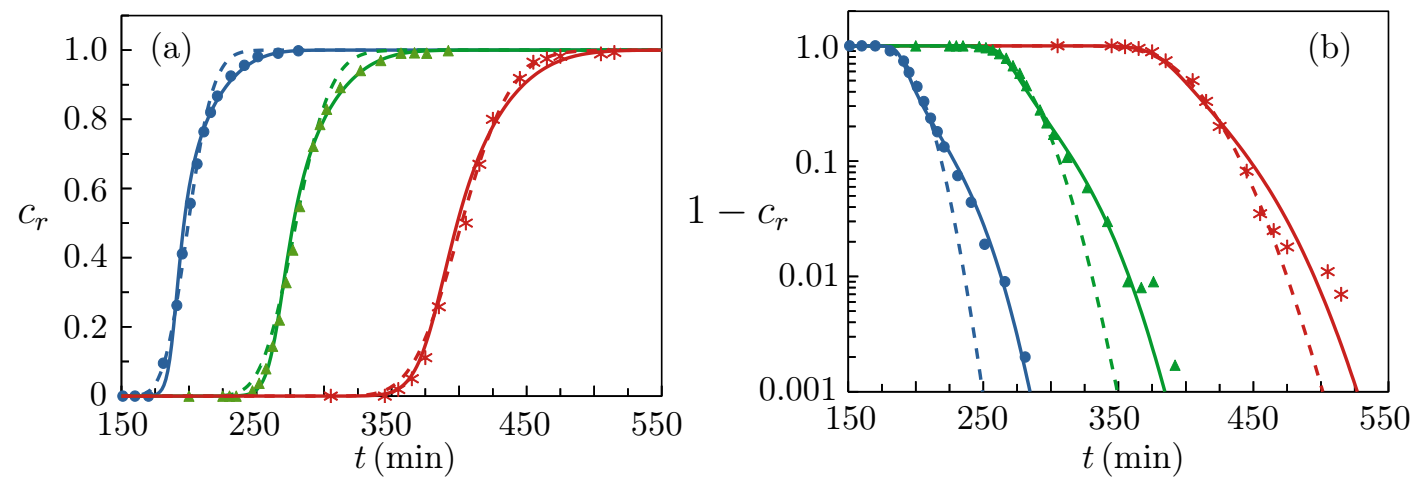

Figure 2: (a) Breakthrough curves for continuous $\mathrm{NaCl}$ injection into a column of sand $(L=$ $\left.0.86 \mathrm{~m}, c_{0}=0.5 \mathrm{~g} / \mathrm{L}\right)$ at three different flow rates. The data are from Levy and Berkowitz [6] at $v=7.4 \mathrm{~m} /$ day (circles), $v=5.3 \mathrm{~m} /$ day (triangles) and $v=3.6 \mathrm{~m} /$ day (stars). (b) The data plotted on a $\log$ scale. The dashed curves in (a) and (b) are from the ADE model (8); the solid curves are from the generalized Burgers equation (16).
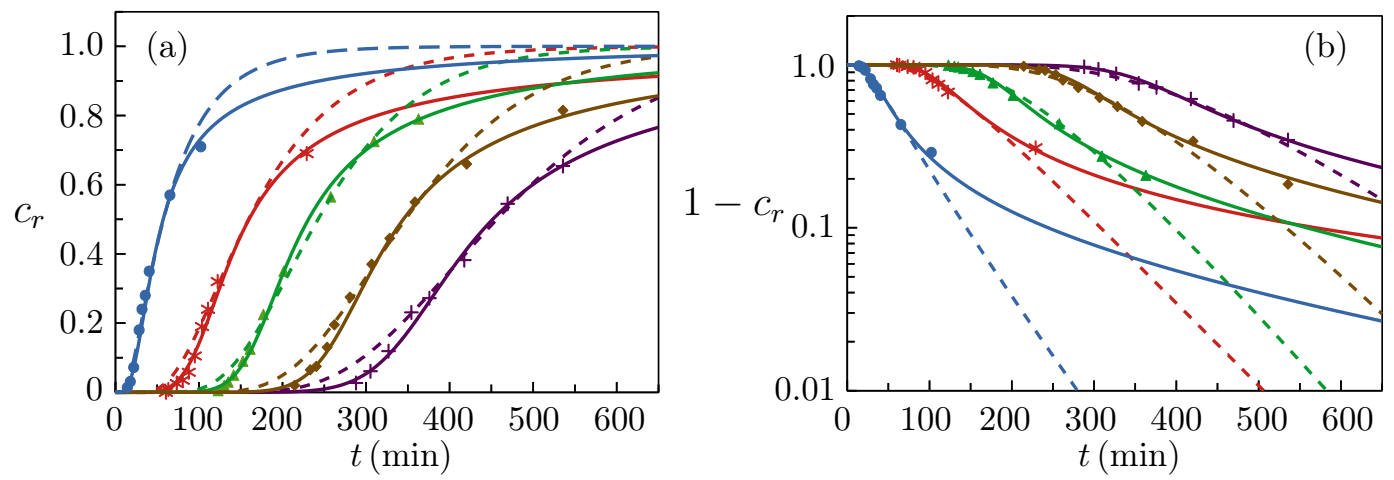

Figure 3: (a) Breakthrough curves measured by Silliman and Simpson $[5,40]$ on a randomly heterogeneous porous medium. The data show BTCs obtained at five different positions along the soil column $(L=0.15 \mathrm{~m}$, blue circles; $0.46 \mathrm{~m}$, red stars; $0.91 \mathrm{~m}$, green triangles; $1.37 \mathrm{~m}$, brown diamonds; $1.83 \mathrm{~m}$, purple plusses). The data in (b) are plotted as $1-c_{r}$ vs $t$ on a log scale. The dashed curves in (a) and (b) are from the ADE model (8); the solid curves are from the generalized Burger's equation (16).

two parameters, $\sigma_{0}$ and $D$. This possibility is supported by data from Silliman and Simpson $[5,40]$ shown in figure 3 . They studied a sandy porous medium composed of 20 mesh sand (diameter $0.7 \mathrm{~mm}$ ), of total length $2.13 \mathrm{~m}$, containing small $(7.5 \mathrm{~cm}$ ) randomly-placed blocks of finer 90 mesh sand (diameter $0.15 \mathrm{~mm}$ ). Figure 3 shows breakthrough curves obtained at several positions $L$ within the porous medium with a constant experimental fluid velocity of $5.8 \mathrm{~m} /$ day [5]. The dashed curves in figure 3 are from the ADE model using the best-fit $v$ and $D$ values shown in Table 1. The solid curves in figure 3 are from (16) using the actual fluid velocity $v=5.8 \mathrm{~m} /$ day, the ADE dispersion coefficients and the $\sigma_{0}$ values shown in Table 1 , along with a single set of reflectivity parameters $a=0.12$ days $/ \mathrm{m}$ and $b=0.053$ days $/ \mathrm{m}$. Similarly to figure 2, good agreement with the data in all cases is obtained using a unique reflectivity function, suggesting that $\alpha_{r}$ is a property of the porous matrix independent of the flow rate or position.

The coefficient $\sigma_{0}$ approached a nearly constant value of -0.2 at larger lengths $L \geq 0.91 \mathrm{~m}$, while $\sigma_{0}$ varied significantly at smaller distances. A possible explanation for the variation in $\sigma_{0}$ is a loss of local equilibrium caused by the presence of the $7.5 \mathrm{~cm}$ low-permeability blocks. As noted by Berkowitz et al. [40], near the entrance to the porous medium the solute plume does not have time to fully explore the low permeability regions, so that the porous medium appears significantly more 


\begin{tabular}{|c|c|c|c|c|c|}
\hline$\overline{L(\mathrm{~m})}$ & 0.15 & 0.46 & 0.91 & 1.37 & 1.83 \\
\hline $\bar{v}_{\text {(ADE) }}(\mathrm{m} /$ day $)$ & $\overline{4.0}$ & $\overline{4.2}$ & 5.4 & 5.7 & 5.8 \\
\hline$D_{\text {(ADE) }}\left(\mathrm{m}^{2} /\right.$ day $)$ & 0.21 & 0.30 & 0.40 & 0.47 & 0.65 \\
\hline$\overline{\sigma_{0}}$ & $\overline{0} . \overline{2} \overline{1}$ & $0 . \overline{12}$ & $-0 . \overline{17}$ & $-0 . \overline{18}$ & $-0 . \overline{20}$ \\
\hline
\end{tabular}

Table 1: Parameter values used to generate the breakthrough curves in figure 3.

heterogeneous on small $(\sim 10 \mathrm{~cm})$ length scales. The model developed here assumes the length scales $L$ at which measurements are taken are much larger than the size of any heterogeneity within the porous medium. It is remarkable that a single set of reflectivity parameters $a$ and $b$ produce a good match to the data even at the shorter length scales. The experimental data were however truncated with only a small portion of the tails available to compare with the model. In Section 4.2 a similar experiment undertaken by Levy and Berkowitz [6] is analyzed in which more data was obtained in the long-time tailing region.

\subsection{Heterogeneous porous media}

In very heterogeneous porous media the simple quadratic form for $\sigma$ in (18) may not be sufficient to reproduce the more complex shape of the breakthrough curves. For example, figure 4 shows breakthrough data obtained using a sample of natural sandstone by Scheidegger [43,44] (volume flowrate $Q=1.73 \mathrm{~cm}^{3} / \mathrm{min}$, cell radius $R=2.54 \mathrm{~cm}$, cell length $L=0.762 \mathrm{~m}$, flow velocity $v=Q / n \pi R^{2}=6.0 \mathrm{~m} /$ day $)$.
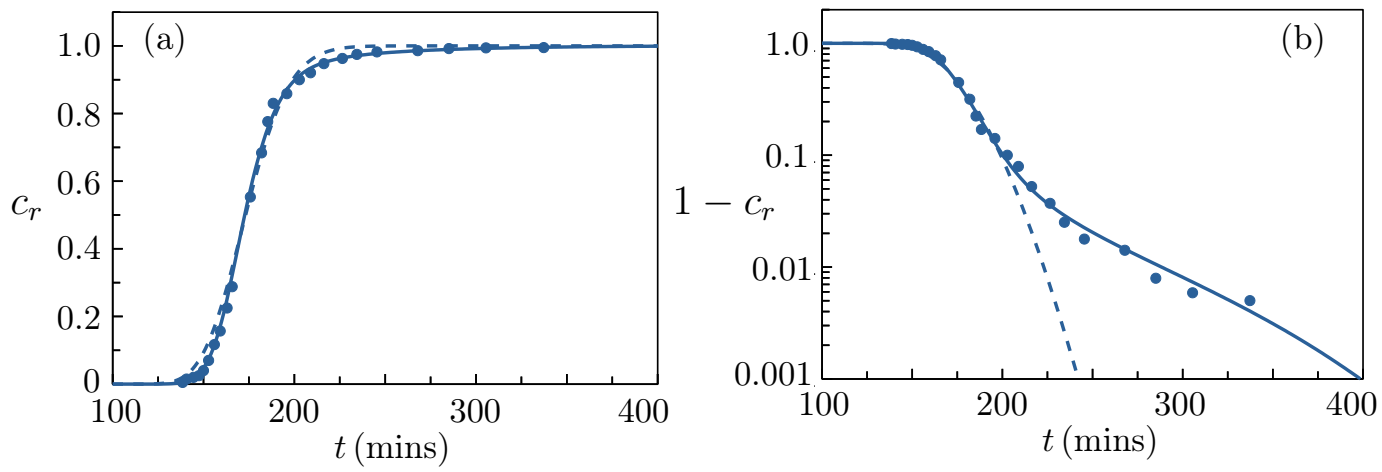

Figure 4: (a) Breakthrough curve for water injection into Berea sandstone of length $L=0.762 \mathrm{~m}$ saturated with a tracer solution. The data (circles) are from Scheidegger [43] (here $c_{r}=1-j$, where $j$ is the relative concentration measured by Scheidegger). The solid curves are from the generalized Burgers equation (16) using (19) for the reflectivity; the dashed curves are from the ADE model (6). (b) The same data plotted as $1-c_{r}$ vs $t$ on a log scale showing the long-time tail.

In this case the breakthrough curve has a broader tail than in figure 1 and a greater departure from the ADE solution; within the natural sandstone there may have been additional effects operating such as multiple porosity regions or a fracture network [44]. A generalization of (18) to account for such effects is $\alpha_{r}=\sum_{i}\left[a_{i}\left(1-c_{r}\right)^{m_{i}}+b_{i} c_{r}^{m_{i}}\right]$, where the sum is over independent types of heterogeneity. Here, assuming the simplest case of a single additional type of heterogeneity, the effects are modelled by the following equation

$$
\alpha_{r}=a\left(1-c_{r}\right)^{2}+b c_{r}^{m}+b\left(1-c_{r}\right)^{m},
$$

which gives a good match to the data using a single additional parameter for the exponent $m$. The power law $c_{r}^{m}$ term in (19) allows for the much more extensive tailing as $c_{r} \rightarrow 1$ within the natural sandstone than occurred within the more uniform sand columns, while the $\left(1-c_{r}\right)^{m}$ term accounts for extended tailing as $c_{r} \rightarrow 0$. The solid curves in figure 4 have been obtained by solving equation (16) with (17) and (19) for $\sigma$, using the dispersion coefficient $D=0.029 \mathrm{~m}^{2} /$ day and reflection 
coefficient parameters $\sigma_{0}=-0.05, a=0.015$ days $/ \mathrm{m}, b=0.002$ days $/ \mathrm{m}$ and $m=40$. The dashed curves are from equation (6) using the parameters $D=0.029 \mathrm{~m}^{2} /$ day and $v=6.3 \mathrm{~m} /$ day.

Levy and Berkowitz [6] performed an experiment on a heterogeneous porous medium designed to emulate the Silliman and Simpson experiment [5]. The data in figure 5 were obtained using a porous medium of length $L=0.86 \mathrm{~m}$ and porosity $n=0.32$ composed of $1.1 \mathrm{~mm}$ diameter sand particles, and containing small $(3 \mathrm{~cm})$ randomly-placed blocks of finer $0.23 \mathrm{~mm}$ diameter sand [6]. Measured breakthrough curves were obtained using three different fluid velocities: $v=7.0 \mathrm{~m} /$ day (blue circles), $v=4.7 \mathrm{~m} /$ day (green triangles) and $v=3.5 \mathrm{~m} /$ day (red plusses). The dashed curves in figure 5 are from the ADE model using the best-fit dispersion coefficient and fluid velocities shown in Table 2. The solid curves are from equation (16) with (19) using the ADE dispersion coefficients, the measured fluid velocities and the reflection coefficient parameters shown in Table 2 .
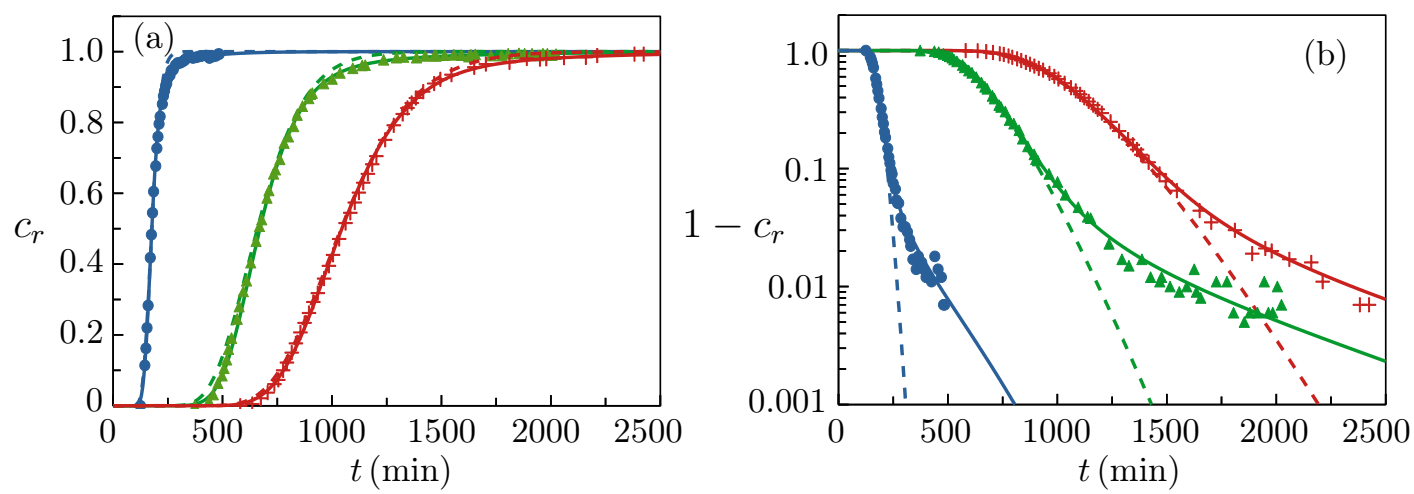

Figure 5: (a) Breakthrough curves for continuous injection of an $\mathrm{NaCl}$ solution into a column of sand $(L=0.86 \mathrm{~m})$ containing randomly-placed low-permeability regions, obtained by Levy and Berkowitz [6] at three different flow rates: $v=7.0 \mathrm{~m} /$ day (blue circles), $v=4.7 \mathrm{~m} /$ day (green triangles) and $v=3.5 \mathrm{~m} /$ day (red plusses). (b) The data in (a) plotted as $1-c_{r} v s t$ on a log scale. The dashed curves in (a) and (b) are from the ADE model (6); the solid curves are from equation (16) using (19) for the reflectivity.

\begin{tabular}{lccc}
\hline$v(\mathrm{~m} /$ day $)$ & 3.5 & 4.7 & 7.0 \\
\hline \hline$v_{(\mathrm{ADE})}(\mathrm{m} /$ day $)$ & 1.2 & 1.9 & 7.0 \\
$D_{(\mathrm{ADE})}\left(\mathrm{m}^{2} /\right.$ day $)$ & 0.032 & 0.055 & 0.10 \\
$\sigma_{0}$ & $0.6 \overline{7}$ & $0.6 \overline{2}$ & 0.014 \\
$a$ (days $/ \mathrm{m})$ & 0.007 & 0.001 & 0.000 \\
$b$ (days $/ \mathrm{m})$ & 0.001 & 50 & 0.0037 \\
$m$ & 50 & & 25 \\
\hline
\end{tabular}

Table 2: Parameter values used to generate the breakthrough curves in figure 5 .

In this case it was not possible to model all of the BTCs using unique dispersivity and reflectivity parameters, in contrast to the more homogeneous porous medium studied by Levy and Berkowitz (figure 2). However, the parameters are closer together for the low velocity green and red data than for the high velocity blue data. A possible explanation is similar to the Silliman and Simpson experiment (Section 4.1); the presence of the low-permeability regions ensures that the solute plume does not have time to fully explore the system and achieve local equilibrium before reaching the measurement position $L$, with the effect enhanced as the fluid velocity increases.

\subsection{Anomalous dispersion in rivers}

Johansson et al. $[45,46]$ performed an experiment in which a dilute solution of tritium was added for a period of $t_{p}=316$ minutes into the Säva stream in Sweden, and breakthrough curves were measured at several locations downstream. The breakthrough curve at $L=2.1 \mathrm{~km}$ for this system 

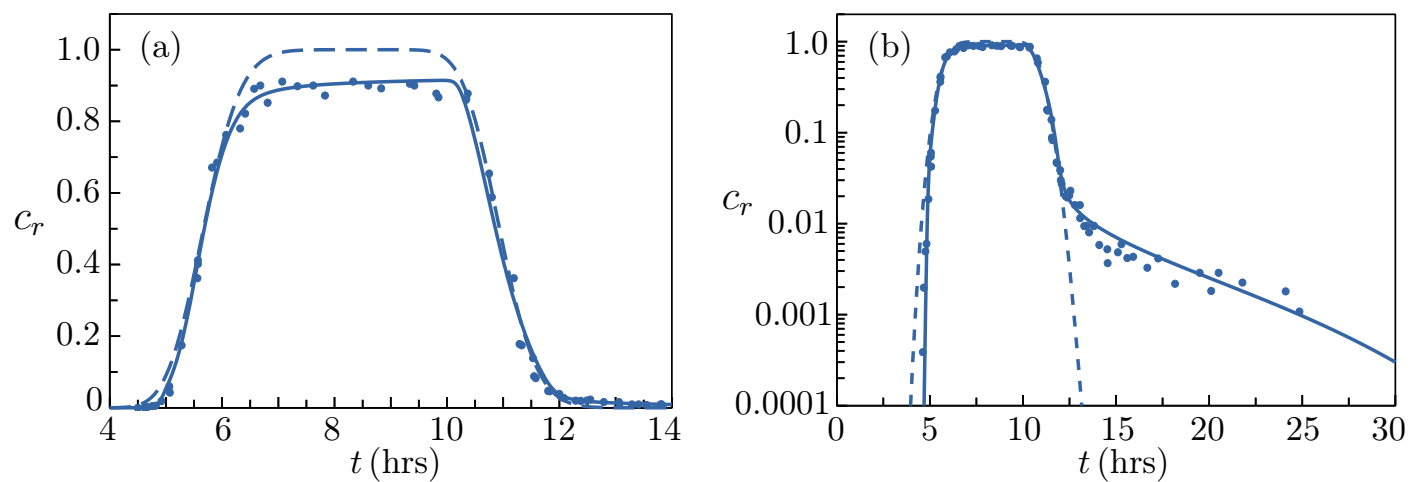

Figure 6: Breakthrough curve for a pulse injection of deuterium into the Säla stream. The data are from Johansson et al [45]. Plotted in (a) are the first $14 \mathrm{hrs}$ of data obtained at a location $L=2.1 \mathrm{~km}$ downstream from the injection point. (b) The data up to $25 \mathrm{hrs}$ plotted as $c_{r}$ vs $t$ on a log scale. The dashed curves are from the ADE model (6); the solid curves are from the generalized Burgers equation (16).

is shown in figure 6 . The data exhibits extensive tailing as $c_{r} \rightarrow 1$ in figure $6 a$ between $6 \mathrm{hrs}$ and $10 \mathrm{hrs}$. Further heavy tailing occurs at later times as $c_{r} \rightarrow 0$, as can be seen when the data for $c_{r}$ is plotted on a $\log$ scale in figure $6 b$. As the average fluid velocity in the stream was not measured, here a best-fit value of $v=9.0 \mathrm{~km} /$ day is used, which is close to the value $v=9.1 \mathrm{~km} /$ day $(0.105 \mathrm{~m} / \mathrm{s})$ used by Johansson et al. [45] in their model of the system. In this case it is not necessary to determine $\sigma_{0}$ for this system. The dashed curves in figure 6 are from the ADE model (6) using the best-fit parameters $v=8.9 \mathrm{~km} /$ day and $D=0.08 \mathrm{~km}^{2} /$ day. The solid curves are from (16) using the ADE dispersion coefficient and the reflectivity parameters $a=0.007$ days $/ \mathrm{km}, b=0.075$ days $/ \mathrm{km}$ and $m=50$. The natural river system is more complex than the laboratory systems, with interactions between the river bed and the bulk of the steam causing the extended tails $[45,46]$. The good fit to the breakthrough curve in figure 6 suggests that it may be possible to approximate the river as a type of high-permeability porous medium with the bed playing a role similar to the low-permeability regions in the Silliman and Simpson [5] and Levy and Berkowitz [6] experiments.

\section{Discussion}

\subsection{Nature of the reflection coefficient}

The reflection coefficient $\sigma$ is a measure of the tendency of the porous medium to reflect the solute particles from the bulk flow, leading to an effective velocity of the solute plume that differs from the average fluid velocity. In low Peclet number flows in membranes and concentrated suspensions, $\sigma$ is caused by surface film phenomena or excluded volume effects $[27,34,47]$; for molecular solutes in inert porous media with relatively large pores these effects are negligible. In high Peclet number flows, however, the viscous segregation coefficient is caused by pore scale eddies, stagnation zones and low permeability regions that temporarily trap solute particles.

When the reflection coefficient is a constant it plays the role of a retardation factor. Dividing by $1-\sigma$, equation (16) can be written as

$$
R_{d} \frac{\partial c}{\partial t}+v \frac{\partial c}{\partial x}=\frac{\partial}{\partial x} D_{d} \frac{\partial c}{\partial x},
$$

where $R_{d}=1 /(1-\sigma)$ is an effective retardation factor and $D_{d}=R_{d} D$ is a rescaled dispersion coefficient. Here the solute retardation is caused by temporary solute trapping within eddies and stagnation zones, rather than by chemical adsorption $[17,48,49]$. It may be possible in future to model $\sigma$ theoretically given the pore and fracture size distribution, for example by using models of 
entrainment in porous-media [50], pore-scale simulations of the fluid flow [8] or fracture network models [51].

\subsection{Conservation of mass at $x=0$.}

An alternative boundary condition often employed is a concentration condition at $x=0$, with $c(0, t)=c_{0}$ replacing $(7 a)[5,21]$. As noted by van Genuchten and Parker [20] this leads at low Peclet numbers to a loss of mass conservation at the boundary. Mass is still conserved within the porous medium itself, but for sufficiently large $D$ some solute is dispersed back into the reservoir at $x<0$. Equation $(7 a)$ on the other hand requires the solute concentration to be discontinuous across the inlet boundary [52], and both boundary conditions neglect dispersion within the reservoir $x<0$. The most appropriate boundary condition at $x=0$ is an ongoing matter of discussion [31,52].

Here equation $(7 a)$ has been employed, as it is frequently used in the literature and is more convenient to impose numerically than the condition of constant $c$ at $x=0$. However, even in this case loss of mass conservation can occur at the boundary for large enough $\sigma$. Parker [31] has suggested that the mass conservation issue can be resolved by ensuring that the dispersivity is zero at the boundary. It seems likely that the reflectivity is also zero at the boundary (at least for molecular solutes in uncharged media with relatively large pores). However, modelling the increase of $\alpha_{d}$ and $\alpha_{r}$ from zero at $x=0$ to their values within the bulk of the porous medium will require additional parameters and is left to future studies.

The expressions (17)-(19) for $\sigma$ have been developed and tested here only for simple initial and boundary conditions (pulse or constant injection conditions). For time dependent boundary conditions or more general initial conditions it is not possible to specify a unique initial concentration $c_{0}$. However, any initial distribution can be approximated as a sequence of independent rectangular pulses, each with its own unique value of $c_{0}$, and the final solution can then be obtained by superposition. In principle the method should therefore work under more general boundary and initial conditions.

\subsection{Relation to stochastic models}

In the stochastic approach to dispersion it is assumed that the classical advection-diffusion equation applies on the pore scale and that the fluid velocity and concentration within a volume element in the system are the sum of their mean values $c, v$ and a stochastic component $c^{\prime}, v^{\prime}$. Applying an averaging procedure then leads to the following stochastic advection-dispersion equation

$$
\frac{\partial c}{\partial t}+v \frac{\partial c}{\partial x}+\frac{\partial\left\langle c^{\prime} v^{\prime}\right\rangle}{\partial x}=D_{0} \frac{\partial^{2} c}{\partial x^{2}}
$$

where $D_{0}$ is the molecular diffusion coefficient and the additional solute flux $\left\langle c^{\prime} v^{\prime}\right\rangle$ is caused by velocity fluctuations $[17,53,54]$. As $c^{\prime}$ and $v^{\prime}$ are unknown the model requires a closure equation to determine the stochastic flux. In the classical 'eddy diffusion' approach it is assumed that

$$
\frac{\partial\left\langle c^{\prime} v^{\prime}\right\rangle}{\partial x}=-\frac{\partial}{\partial x} D_{e} \frac{\partial c}{\partial x}
$$

where $D_{e}=\alpha_{d} v$ is the hydrodynamic dispersion coefficient $[17,55]$. This assumption is convenient as it allows (21) to take a deterministic form equivalent to (6); however the model cannot describe anomalous breakthrough curves involving nonGaussian dispersion $[17,56]$. In the stochastic advection-diffusion approach it is instead assumed that $\left\langle c^{\prime} v^{\prime}\right\rangle$ is determined by the solution to a differential equation governing the fluctuating variable $c^{\prime}$, given an assumed or prescribed distribution of $v^{\prime}[2,54]$. In the present model the closure relation is of the form

$$
\frac{\partial\left\langle c^{\prime} v^{\prime}\right\rangle}{\partial x}=-v \frac{\partial \sigma c}{\partial x}-\frac{\partial}{\partial x} D_{e} \frac{\partial c}{\partial x} .
$$

Like (22), equation (23) is deterministic, and it is the nonlinear reflection coefficient $\sigma(c, v)$ that allows for anomalous breakthrough curves to be simulated. It is hoped in future to relate the parameters determining the reflection coefficient to properties of the porous medium like the pore and fracture size distributions. 


\section{Conclusion}

A theory of anomalous dispersion has been developed by taking the high Peclet number limit of the equations governing cross-diffusion in concentrated suspensions and porous media. This leads to coupling terms between the Darcy flux and the tracer dispersion flux. For high Peclet number flows in a rigid porous matrix the model yields a modified advection-dispersion equation containing a reflection coefficient in the advection term. Allowing the reflection coefficient to depend on the flow rate and relative tracer concentration leads to good agreement with experimental breakthrough curves. Advantages of the model are its relative simplicity and conformity to the basic mathematical structure of classical continuum mechanics. In addition the model uses the real fluid velocity, in contrast to the ADE model in which the velocity is treated as an adjustable parameter. The expression used for the reflection coefficient contains a heterogeneity parameter, the reflectivity, that appears to be a unique property of the porous matrix; it may in future be possible to relate the reflectivity to physical properties of the porous medium.

\section{Acknowledgements}

I thank the Lord Jesus, for His love and merciful kindness, for restoring my soul, and for allowing me to study His beautiful creation. Believe on the Lord Jesus Christ and thou shalt be saved, and thy house. Acts 16:31. For the Son of man is not come to destroy men's lives, but to save them. Luke 9:56.

\section{References}

[1] Brian Berkowitz, Andrea Cortis, Marco Dentz, and Harvey Scher. Modeling non-Fickian transport in geological formations as a continuous time random walk. Reviews of Geophysics, 44, RG2003 1-49, 2006.

[2] Shlomo P. Neuman and Daniel M. Tartakovsky. Perspective on theories of non-Fickian transport in heterogeneous media. Advances in Water Resources, 32, 670 - 680, 2009.

[3] M. Dentz, M. Icardi, and J. J. Hidalgo. Mechanisms of dispersion in a porous medium. Journal of Fluid Mechanics, 841, 851-882, 2018.

[4] XR. Yang and Y. Wang. Ubiquity of anomalous transport in porous media: Numerical evidence, continuous time random walk modelling, and hydrodynamic interpretation. Sci. Rep., 9, 4601, 2019.

[5] S. E. Silliman and E. S. Simpson. Laboratory evidence of the scale effect in dispersion of solutes in porous media. Water Resources Research, 23, 1667-1673, 1987.

[6] Melissa Levy and Brian Berkowitz. Measurement and analysis of non-Fickian dispersion in heterogeneous porous media. Journal of Contaminant Hydrology, 64, 203 - 226, 2003.

[7] Xiaoxian Zhang and Mouchao Lv. Persistence of anomalous dispersion in uniform porous media demonstrated by pore-scale simulations. Water Resources Research, 43, W07437, 2007.

[8] Pietro de Anna, Bryan Quaife, George Biros, and Ruben Juanes. Prediction of the low-velocity distribution from the pore structure in simple porous media. Phys. Rev. Fluids, 2, 124103, 2017.

[9] X.-R. Yang and Y. Wang. Ubiquity of anomalous transport in porous media: Numerical evidence, continuous time random walk modelling, and hydrodynamic interpretation. Sci. Rep., 9, 4601, 2019.

[10] Brian Berkowitz and Harvey Scher. The role of probabilistic approaches to transport theory in heterogeneous media. Transport in Porous Media, 42, 1573-1634, 2001. 
[11] Yong Zhang, David A. Benson, Mark M. Meerschaert, and Eric M. LaBolle. Space-fractional advection-dispersion equations with variable parameters: Diverse formulas, numerical solutions, and application to the Macrodispersion Experiment site data. Water Resources Research, 43, W05439, 2007.

[12] B. Ghanbarian, A. G. Hunt, R. P. Ewing, and M. Sahimi. Tortuosity in porous media: A critical review. Soil Science Society of America Journal, 77, 1461-1477, 2013.

[13] Donald L. Koch and John F. Brady. Anomalous diffusion in heterogeneous porous media. The Physics of Fluids, 31, 965-973, 1988.

[14] Jose A. Ferreira and Luis Pinto. An integro-differential model for non-Fickian tracer transport in porous media: validation and numerical simulation. Mathematical Methods in the Applied Sciences, 39, 4736-4749, 2016.

[15] A. Fiori, A. Zarlenga, H. Gotovac, I. Jankovic, E. Volpi, V. Cvetkovic, and G. Dagan. Advective transport in heterogeneous aquifers: Are proxy models predictive? Water Resources Research, 51, 9577-9594, 2015.

[16] Scott K. Hansen and Brian Berkowitz. Aurora: A non-Fickian (and Fickian) particle tracking package for modeling groundwater contaminant transport with MODFLOW. Environmental Modelling \& Software, 134, 104871, 2020.

[17] J. Bear. Dynamics of Fluids in Porous Media. Elsevier, NY, 1972.

[18] Garrison Sposito, Vijay K Gupta, and R.N Bhattacharya. Foundation theories of solute transport in porous media: a critical review. Advances in Water Resources, 2, 59-68, 1979.

[19] John F. Pickens and Gerald E. Grisak. Modeling of scale-dependent dispersion in hydrogeologic systems. Water Resources Research, 17, 1701-1711, 1981.

[20] M. Th. van Genuchten and J.C. Parker. Boundary conditions for displacement experiments through short laboratory soil columns. Soil Sci. Soc. Am. J., 48, 703-708, 1984.

[21] Charles D. Shackelford. Critical concepts for column testing. Journal of Geotechnical Engineering, 120, 1804-1828, 1994.

[22] Fredrick T. Lindstrom, Rizwanul. Haque, Virgil H. Freed, and Larry. Boersma. The movement of some herbicides in soils. Linear diffusion and convection of chemicals in soils. Environmental Science \& Technology, 1, 561-565, 1967.

[23] Andrea Cortis, Youjian Chen, Harvey Scher, and Brian Berkowitz. Quantitative characterization of pore-scale disorder effects on transport in "homogeneous" granular media. Phys. Rev. E, 70, 041108, 2004.

[24] S. S. L. Peppin, J. A. W. Elliott, and M. G. Worster. Pressure and relative motion in colloidal suspensions. Physics of Fluids, 17(5), 053301, 2005.

[25] S. S. L. Peppin, J. A. W. Elliott, and M. G. Worster. Solidification of colloidal suspensions. J. Fluid Mech., 554, 147-166, 2006.

[26] S. S. L. Peppin. On diffusion and permeation. Journal of Non-Equilibrium Thermodynamics, 34, 355-369, 2009.

[27] S. S. L. Peppin. Theory of tracer diffusion in concentrated hard-sphere suspensions. J. Fluid Mech., 870, 1105-1126, 2019.

[28] S. S. L. Peppin. Anomalous tracer diffusion in hard-sphere suspensions. engrXiv, https://engrxiv.org/nm95k, 2021.

[29] G. K. Batchelor. Note on the Onsager symmetry of the kinetic coefficients for sedimentation and diffusion in a dilute bidispersion. Journal of Fluid Mechanics, 171, 509-517, 1986. 
[30] O. Annunziata. On the role of solute solvation and excluded-volume interactions in coupled diffusion. The Journal of Physical Chemistry B, 112, 11968-11975, 2008.

[31] Jack C. Parker and Ungtae Kim. An upscaled approach for transport in media with extended tailing due to back-diffusion using analytical and numerical solutions of the advection dispersion equation. Journal of Contaminant Hydrology, 182, 157-172, 2015.

[32] A. Puyguiraud, P. Gouze, and M. Dentz. Is there a representative elementary volume for anomalous dispersion? Transp. Porous Med., 131, 767-778, 2020.

[33] K. S. Spiegler and O. Kedem. Thermodynamics of hyperfiltration (reverse osmosis): Criteria for efficient membranes. Desalination, 1, 311-326, 1966.

[34] A. Katzir-Katchalsky and P. F. Curran. Nonequilibrium Thermodynamics in Biophysics. Harvard University Press, Boston, 1965.

[35] Andrea Dominijanni and Mario Manassero. Modelling the swelling and osmotic properties of clay soils. part I: The phenomenological approach. International Journal of Engineering Science, 51, 32-50, 2012.

[36] M. A. Malusis, C. D. Shackelford, and J. E. Maneval. Critical review of coupled flux formulations for clay membranes based on nonequilibrium thermodynamics. Journal of Contaminant Hydrology, 138, 40-59, 2012.

[37] V. Vanaja and P. L. Sachdev. Asymptotic solutions of a generalized Burgers equation. Quarterly of Applied Mathematics, 50, 627-640, 1992.

[38] A. S. Tersenov. On the generalized Burgers equation. Nonlinear Differ. Equ. Appl., 17, 437-452, 2010.

[39] Mayur P. Bonkile, Ashish Awasthi, C. Lakshmi, Vijitha Mukundan, and V. S. Aswin. A systematic literature review of Burgers equation with recent advances. The Journal of Chemical Physics, 90, 1-21, 2018.

[40] Brian Berkowitz, Harvey Scher, and Stephen E. Silliman. Anomalous transport in laboratoryscale, heterogeneous porous media. Water Resources Research, 36, 149-158, 2000.

[41] J.M. Burgers. A mathematical model illustrating the theory of turbulence. Advances in Applied Mechanics, 1, 171-199, 1948.

[42] W. E. Scheisser and G. W. Griffiths. A Compendium of Partial Differential Equation Models: Method of Lines Analysis with Matlab. Cambridge University Press, U.K., 2009.

[43] A. E. Scheidegger. An evaluation of the accuracy of the diffusivity equation for describing miscible displacement in porous media. In Proceedings of the Conference on Theory of Fluid Flow in Porous Media, pages 101-116, Norman, Oklahoma, 1959.

[44] Andrea Cortis and Brian Berkowitz. Anomalous transport in "classical" soil and sand columns. Soil Sci. Soc. Am. J., 68, 1539-1548, 2004.

[45] Håkan Johansson, Karin Jonsson, K. Jonas Forsman, and Anders Wärman. Retention of conservative and sorptive solutes in streams - simultaneous tracer experiments. Science of The Total Environment, 266, 229-238, 2001.

[46] Karin Jonsson, Håkan Johansson, and Anders Wärman. Hyporheic exchange of reactive and conservative solutes in streams - tracer methodology and model interpretation. Journal of Hydrology, 278, 153-171, 2003.

[47] J. L. Anderson and J. A. Quinn. Restricted transport in small pores: A model for steric exclusion and hindered particle motion. Biophysical Journal, 14, 130-150, 1974.

[48] R. Allan Freeze and John A. Cherry. Groundwater. Prentice Hall, New Jersey, 1979. 
[49] Scott K. Hansen and Velimir V. Vesselinov. Local equilibrium and retardation revisited. Groundwater, 56, 109-117, 2018.

[50] Chunendra K. Sahu and Jerome A. Neufeld. Dispersive entrainment into gravity currents in porous media. Journal of Fluid Mechanics, 886, A5, 2020.

[51] M. Sahimi. Flow and Transport in Porous Media and Fractured Rock: From Classical Methods to Modern Approaches, Second Edition. Wiley-VCH, Germany, 2012.

[52] J.-Y. Parlange, D. A. Barry, and J. L. Starr. Comments on "Boundary conditions for displacement experiments through short laboratory soil columns". Soil Sci. Soc. Am. J., 49, $1325,1985$.

[53] P. J. Roberts and D. R. Webster. Turbulent diffusion. In H. H. Shen et al., editor, Environmental Fluid Mechanics: Theories and Applications, pages 7-47. ASCE Publications, USA, 2002 .

[54] Eric Morales-Casique, Shlomo P. Neuman, and Alberto Guadagnini. Nonlocal and localized analyses of nonreactive solute transport in bounded randomly heterogeneous porous media: Computational analysis. Advances in Water Resources, 29, 1399-1418, 2006.

[55] Geoffrey Ingram Taylor. Eddy motion in the atmosphere. Philosophical Transactions of the Royal Society of London. Series A, 215, 1-26, 1915.

[56] Benoit Cushman-Roisin. Turbulent dispersion. In J. S. Fernando, editor, Handbook of Environmental Fluid Dynamics, Volume One, pages 263-271. CRC Press/Taylor \& Francis, USA, 2013. 